\title{
Broadband Terahertz Time-Domain and Low-Frequency Raman Spectroscopy of Crystalline and Glassy Pharmaceuticals
}

Seiji Kojima*,†, Tatsuya Mori ${ }^{\dagger}$, Tomohiko Shibata and Yukiko Kobayashi

Division of Materials Science, University of Tsukuba, Tsukuba, Ibaraki 305-8573, Japan

†These authors contributed equally to this work

\begin{abstract}
The applications of the broadband Terahertz Time-Domain Spectroscopy (THz-TDS) and the low-frequency Raman scattering spectroscopy to glassy and crystalline pharmaceuticals were reviewed. The real and imaginary parts of a complex dielectric constant were measured by the transmission of THz-TDS using a pure pellet without mixing polyethylene in the frequency range from 0.2 to $6.5 \mathrm{THz}$. The low-frequency Raman spectra were measured down to $0.3 \mathrm{THz}$ using a double-grating spectrometer. In crystalline indomethacin, indapamide, and racemic ketoprofen, the clear difference in peak frequencies was observed between $\mathrm{THz}$ dielectric and low-frequency Raman scattering spectra. It can be attributed to the mutual exclusion principle between Raman and IR activities of optical vibrational modes in centrosymmetric crystals. In glassy indomethacin the broad peak at $3.0 \mathrm{THz}$ in the IR spectrum is attributed to the infrared active intermolecular vibrational mode of the hydrogen bonded cyclic dimers, which is centrosymmetric. The boson peak is the well-known low-energy excitation reflecting vibrational density of states in glassy or amorphous materials. The boson peaks of glassy indomethacin were clearly observed at about $0.3 \mathrm{THz}$ and $0.5 \mathrm{THz}$ in THz-TDS and Raman scattering spectra, respectively. The difference in peak frequencies is attributed to the different frequency dependence between IR-vibration and Raman-vibration coupling constants.
\end{abstract}

\section{Introduction}

In current studies of pharmaceuticals, it is important to characterize not only polymorphic natures in crystalline states but also amorphous or glassy nonequilibrium states. The vibrational spectroscopy is very sensitive for the differences in crystallographic structure and crystal symmetry. For noncrystalline materials, namely amorphous and glassy materials, it is also a powerful tool to elucidate the intermediate structure and dynamical properties related to bond strength and molecular interaction.

The far-IR spectroscopy has been extensively applied to various kinds of crystalline and glassy materials to investigate low-energy excitations such as soft modes of structural phase transitions, polaritons, excitons, boson peaks, etc., while most far-IR studies reported only the absorbance or transmittance, and the reliable reports on the real and imaginary parts of dielectric constants were rare. However, the recent progress in the coherent terahertz generation using a femtosecond pulse laser enables the unique determination of a complex dielectric constant without the Kramers-Kronig transformation, and terahertz time-domain spectroscopy (THz-TDS) has attracted much attention. However, in the most of the THz-TDS measurements, the high frequency limit is below $3 \mathrm{THz},\left(100 \mathrm{~cm}^{-1}\right)$ [1-3]. Very recently the widely tunable monochromatic Cherenkov phase-matched $\mathrm{THz}$ wave generator was developed using nonlinear optic crystals of ferroelectric $\mathrm{MgO}$ doped $\mathrm{LiNbO}_{3}$ [4]. The frequency range of $\mathrm{THz}-$ TDS has been markedly extended up to $6.5 \mathrm{THz}$ by the broadband $\mathrm{THz}$ generator. Moreover, the improvement of the rapid scan technique enables to acquire a high quality $\mathrm{THz}$ spectrum using the high-speed asynchronous optical sampling (AOS) technique.

In this review, transmission broadband $\mathrm{THz}$ spectra in the frequency range from 0.2 to $6.5 \mathrm{THz}$ were studied in glassy and crystalline states of polymorphic and polymorphic pharmaceuticals by using aTHz-TDS equipment with a Cherenkov type $\mathrm{THz}$ generator and the high-speed AOS technique [5]. For the comparison of THz-TDS spectra with Raman scattering spectra, the low-frequency Raman spectra were also accurately measured by a double-grating monochromator, and the exclusion principle of IR and Raman selection rules of centro-symmetric crystals was discussed.

\section{Broadband Terahertz Time-Domain Spectroscopy and Low-Frequency Raman Scattering}

$\mathrm{THz}$ transmission spectra were measured in the frequency range from 0.2 to $6.5 \mathrm{THz}$ using THz-TDS equipments (RT-10000, Tochigi Nikon Co., TAS7500SU, Advantest Co.) with a Cherenkov type $\mathrm{THz}$ generator and the high-speed AOS technique. The comparison of $\mathrm{THz}$ time-domain waveforms and the frequency-domain radiation power between a conventional low-temperature grown GaAs dipole photoconductive antenna and a Cherenkov type $\mathrm{THz}$ generator is shown in Figure 1 (a) and (b), respectively. The photoconductive antenna has a broad peak at about $0.7 \mathrm{THz}$, while the peak frequency of a Cherenkov type is around $2.5 \mathrm{THz}$ and very broad. Therefore, the latter type generator enables us to observe a broadband spectrum up to $6.5 \mathrm{THz}[5]$.

Most of conventional compact Raman spectrometers consist of a single grating-monochromator and a notch filter to reduce the strong elastic scattering. The low-frequency limit is usually around $100 \mathrm{~cm}^{-1}$ $(3.3 \mathrm{THz})$. Recent development of the super-notch filter for microRaman system enables the measurement down to $5 \mathrm{~cm}^{-1}$ using the combination of few super-notch filters, and it is possible to observe a low-frequency sharp peak such as the narrow and intense Raman peak

*Corresponding author: Seiji Kojima and Tatsuya Mori, Division of Materials Science University of Tsukuba, Tsukuba, Ibaraki, Japan, Tel: 305-8573; Email: kojima@ bk.tsukuba.ac.jp

Received July 28, 2015; Accepted August 11, 2015; Published August 13, 2015

Citation: Kojima S, Mori T, Shibata T, Kobayashi Y (2015) Broadband Terahertz Time-Domain and Low-Frequency Raman Spectroscopy of Crystalline and Glassy Pharmaceuticals. Pharm Anal Acta 6: 401. doi:10.4172/21532435.1000401

Copyright: ( 2015 Kojima S, et al. This is an open-access article distributed under the terms of the Creative Commons Attribution License, which permits unrestricted use, distribution, and reproduction in any medium, provided the original author and source are credited. 

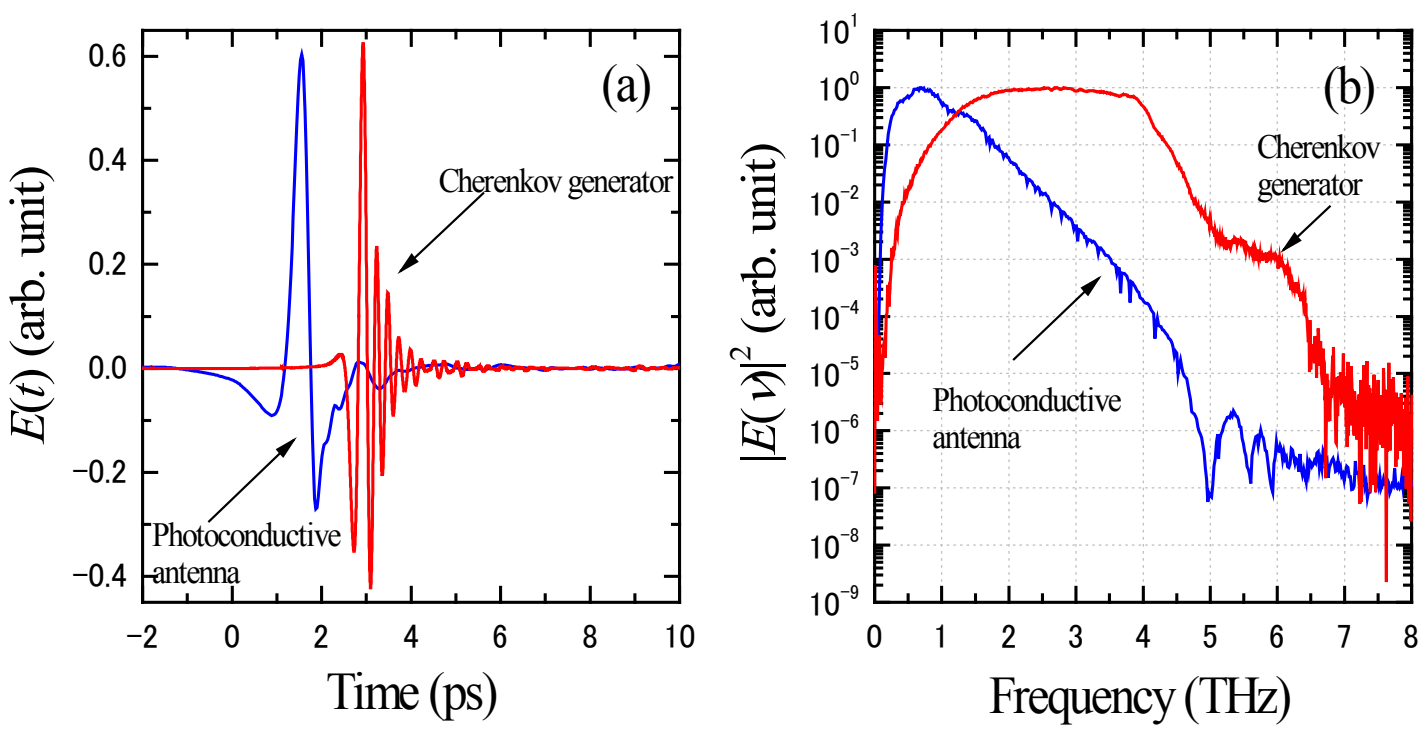

Figure 1: Comparison of (a) time-domain waveforms and (b) frequency-domain $\mathrm{THz}$ power spectra between a Cherenkov type THz generator and a dipole photoconductive antenna.

of L-cystine at $9.6 \mathrm{~cm}^{-1}$. However, a notch filter causes the distortion of Raman line shape in the vicinity of wavelength of an incident beam. Therefore, the conventional Raman system using a notch filter is not suitable for the observation of the broad peak of an anharmonic mode and the quasi-elastic scattering of a relaxation mode in the very low frequency region below $1 \mathrm{THz}$. Especially, amorphous or glassy materials show various broad responses such as $\beta$-relaxation and boson peak [6]. In addition, the spectral resolution of a compact Raman spectrometer is much lower than that of current THz-TDS systems. Consequently, for the comparison of accurate THz-TDS spectra with Raman spectra, especially low-frequency broad peaks, the use of a traditional large double-grating or triple-grating monochromator with a single channel detector such as a photomultiplier tube with very low dark counts is necessary to determine a reliable Raman line shape without any distortion.

Raman spectra have been measured using a double-grating monochromator of additive dispersion (Horiba-Jobin Yvon, U1000) with the spectral resolution of 1-2 $\mathrm{cm}^{-1}$. The light from a green YAG laser with wavelength $532 \mathrm{~nm}$ and power of $100 \mathrm{~mW}$ was focused into a sample through a lens with a focal length of $200 \mathrm{~mm}$. The output signals from the spectrometer were detected by the photon-counting system with a photomultiplier (Hamamatsu Photonics, R464S) [7].

\section{Comparison between Thz-TDS and Raman scattering spectra in pharmaceuticals}

The most of pharmaceuticals are polymorphic and polyamorphic originated from multi-basin structure in the potential energy landscape. The energy states of these basins are nearly degenerate, and the transition among basins can be easily occurred by the change of temperature, pressure, and other chemical conditions. The dynamical properties in the $\mathrm{THz}$ frequency range are very sensitive for the difference in structure and symmetry among polymorphic and polyamorphic states. Therefore, the investigation on each state using
THz-TDS and low-frequency Raman scattering is very important to give new insights into polymorphic and polyamorphic nature of pharmaceuticals.

\section{Crystalline indapamide}

Indapamide (IND) is 4-chloro-N-(2-methyl-2,3-dihydroindol-1yl)-3-sulfamoylbenzamide with the chemical formula, $\mathrm{C}_{16} \mathrm{H}_{16} \mathrm{ClN}_{3} \mathrm{O}_{3} \mathrm{~S}$. In the treatment of hypertension, the commercial type IND exerts spasmolytic effects on blood vessels, consequently reducing the blood pressure. It is an organic glass former, and it undergoes a liquid-glass transition at a relatively high glass transition temperature, $\mathrm{Tg}=376 \mathrm{~K}$ [8]. The unit cell of a crystalline state of commercial type IND contains four IND molecules $(Z=4)$, and those molecules form two types of cavities. The cavity encapsulates water molecules in non-stoichiometric. The water molecules are weakly bounded in these cavities, and can easily come out and in from the IND crystalline framework $[9,10]$. The commercial type crystalline IND with the purity $>99 \%$ was purchased from Sigma-Aldrich Corp., and was used without further purification. Figure 2 (a) shows the real part of the complex dielectric constants, $\varepsilon^{\prime}(v)$, of the commercial type crystalline state of IND. Figure 2(b) shows the comparison of the imaginary parts between the dielectric constant , $\varepsilon "(v)$, and the Raman susceptibility, $\chi{ }^{\prime \prime}(v)[11]$.

In the observed frequency range, several phonon peaks were observed. These modes can be attributed to inter- or intra-molecular vibrational modes in the crystalline state. The discrepancy of the phonon peak frequencies was clearly observed between $\varepsilon$ " $(v)$ and $\chi$ ” $(v)$ as shown in Figure 2(b). For a centrosymmetric crystal, of which point group of a crystal structure includes the center of symmetry, the mutual exclusion principle holds between Raman and IR activities. It means that all Raman active modes are IR inactive, and vice versa [12]. Consequently, it is concluded that the point symmetry of the crystalline IND has a center of symmetry, and this result is consistent with the reported crystal structure determined by the X-ray diffraction [10]. 


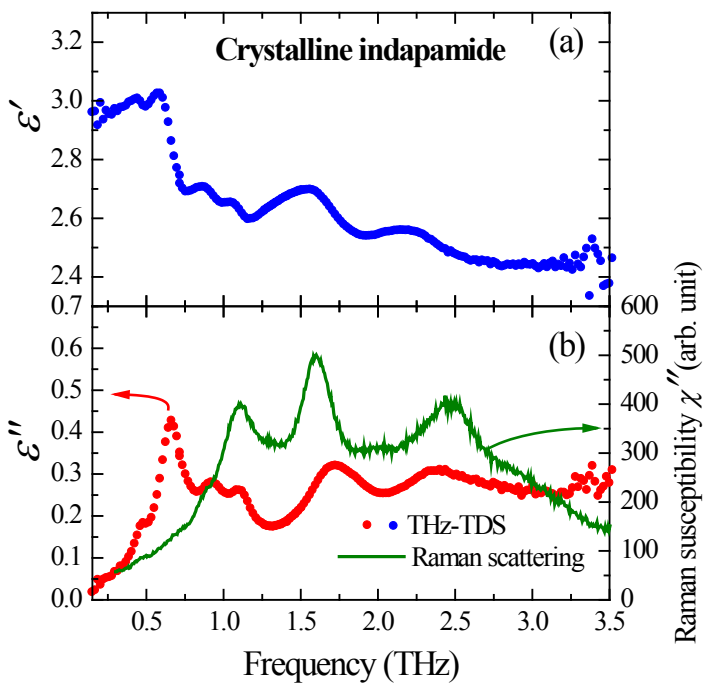

Figure 2: (a) Real part of the complex dielectric constant, $\varepsilon^{\prime}(\mathrm{v})$, and (b) the imaginary parts of the complex dielectric constant, $\varepsilon$ "(v), and the Raman susceptibility, $X "(v)$, of the commercial type crystalline state of indapamide at room temperature.

\section{Crystalline racemic ketoprofen}

Racemic ketoprofen, (RS)-2-(3-benzoylphenyl) propanoic acid with chemical formula $\mathrm{C}_{16} \mathrm{H}_{14} \mathrm{O}_{3}$ is a nonsteroidal anti-inflammatory drug (NSAID) used for the treatment of inflammation and pain. It contains a chiral centre at the $\alpha$-carbon to the carboxyl function and $\mathrm{R}(-)$ and $\mathrm{S}(+)$ enantiomeric forms are available [13]. Racemic ketoprofen with the purity $\geq 98 \%$ was purchased from Sigma-Aldrich. Ketoprofen is polymorphic, and nine conformers were known [14]. A $\mathrm{THz}$ transmission spectrum was measured using a pure pellet without mixing polyethylene, and the real and imaginary parts of complex dielectric constant is shown in Figure 3(a) and (b), respectively. The Raman scattering spectrum of the present sample was also measured as shown in Figure 3(b) The Raman spectrum is assigned to "conformer A" according to the experimental and calculated mode frequencies reported in ref [14].

Ketoprofen exhibits intense low-frequency peaks in the spectra of imaginary parts of Raman and dielectric susceptibilities. These modes originate from the intermolecular vibration modes in a crystalline state. We decomposed the measured spectra using multi-peak fitting procedure. Peaks are described by spectral functions of dumped harmonic oscillators (DHO) to determine the mode frequency and damping of each mode. From these results and the reported results of DFT calculation and vibrational assignment [14], it is suggested that one possible origin of intense low-frequency peaks such as $1.38,2.04$ and $2.91 \mathrm{THz}$ in a Raman scattering spectrum is the vibrations relate to hydrogen bonded ketoprofen dimers in the crystal structure [15].

\section{Crystalline indomethacin}

The indomethacin, 1-[19]-5-methoxy-2-methylindole-3-acetic acid with chemical formula $\mathrm{C}_{19} \mathrm{H}_{16} \mathrm{ClNO}_{4}$, (IMC) is a nonsteroidal anti-inflammatory drug (NSAID) used for the treatment of pain. The crystalline $\gamma$-form $(\gamma$-IMC) with the purity $\geq 99 \%$ was purchased from Sigma-Aldrich and used without further purification. Up to the present several papers reported $\mathrm{THz}$ absorbance spectra of glassy and crystalline IMC [16-18]. In different from the previous $\mathrm{THz}$ studies, the pellet mixed with polyethylene powder was not used. All spectra were measured using a thin plate or a pressed pure pellet without mixing other solid powders such as polyethylene, because such a preparation is very important for the accurate determination of the real and imaginary parts of a complex dielectric constant. Since the absorption in the $\mathrm{THz}$ frequency range is not strong, the real and imaginary parts of a dielectric constant were determined by the transmission measurement. Figure 4(a) and (b) show the observed results of the real and imaginary parts of complex dielectric constant spectra and a low-frequency Raman scattering spectrum of $\gamma$-IMC.

As shown in Figure 4(a) and (b), the $\gamma$-IMC exhibits several phonon peaks in the $\varepsilon$ " $(v)$ and $\chi$ ” $(v)$ spectra. In $\gamma$-IMC, intermolecular vibration modes as well as intramolecular modes are expected in the measured $\mathrm{THz}$ frequency range. The difference between the $\varepsilon "(v)$ and the $\chi$ " $(v)$ was clearly observed below $6.5 \mathrm{THz}$. It was reported from the X-ray diffraction [19] that the crystal structure of the $\gamma$-IMC belongs to the centrosymmetric triclinic space group $\mathrm{P} 1$ with $\mathrm{Z}=2$ and that the carboxylic acid groups hydrogen bond to form cyclic dimers. As a consequence, it can be reasonably stated that the exclusive principle of the IR and Raman selection rules holds for the $\gamma$-IMC [20].

\section{Glassy indomethacin}

Pellets or thin plates of glassy samples were made by the meltquenching method. Figure 5(a) and (b) show the real and imaginary parts of the dielectric constants for the glassy IMC determined in the frequency range of $0.2-6.5 \mathrm{THz}$ at $296 \mathrm{~K}$. The strong and very broad resonant-type dielectric dispersion can be clearly seen at $3.0 \mathrm{THz}$, while weak contributions could be seen at around 1.0, 4.8 and 6.0 THz. There are no sharp absorption peaks in the spectrum of the glassy IMC in comparison with Figure 4(b) of the $\gamma$-IMC. In contrast to the spectra of normal liquids below 1.0 THz [21-23], $\varepsilon$ " $(v)$ of the glassy IMC does not show any tail of the dielectric relaxation processes. This is mainly because the relaxation processes shift to a lower frequency range at the temperatures far below the liquid-glass transition temperature [24,25]. Figure 5(b) shows the Raman susceptibility $\chi$ "( $(v)$ of the glassy IMC. The shape of the Raman susceptibility spectrum also shows a broad and asymmetric peak around $1.2 \mathrm{THz}$ and weak peaks around 4.5 and $6.0 \mathrm{THz}$. We found that the line shape of the Raman spectrum above $4.0 \mathrm{THz}$ resembles the dielectric spectrum. However, a very broad and strong absorption peak at $3.0 \mathrm{THz}$ was observed only in the $\varepsilon "(v)$ spectrum, but not in the $\chi^{\prime \prime}(v)$ spectrum. Two possible origins for this behavior around $3.0 \mathrm{THz}$ can be proposed as followings: (1) The Raman scattering intensity of the vibrational mode was not very strong and hidden by the intense and broad boson peak. (2) The mode is essentially IR active and Raman inactive.

A cyclic dimer has a center of symmetry and the mutual exclusion rule can hold. In the low-frequency range, previous studies of liquid carboxylic acids reported that a broad vibrational mode around 4.5 $\mathrm{THz}$ was observed by far-infrared spectroscopy and assigned as an IR active intermolecular vibrational mode between the hydrogen bonded cyclic dimers [26,27]. The existence of hydrogen bonded cyclic dimers in a glassy state was suggested by the observation of the infrared active intermolecular vibrational mode of a hydrogen bonded cyclic dimer as a broad peak at $3.0 \mathrm{THz}$ in the IR spectrum.

The most of amorphous or glassy materials show the common nature of the low-energy excitations. It was known as a boson peak and has been observed as a broad and asymmetric peak at few $\mathrm{THz}$ by Raman scattering, far-infrared spectroscopy, neutron and X-ray 
inelastic scattering $[28,29]$. It reflects the vibrational density of states (VDOS), $g(v)$, divided by the square of frequency $v$.

In Far IR spectroscopy, the boson peak is given by,

$\frac{2 \pi \cdot \varepsilon^{\prime \prime}(v)}{c . \eta^{\prime}(v) v}=\frac{\alpha(v)}{v^{2}}=C_{I R}(v) \cdot \frac{g(v)}{v^{2}}$

where $n^{\prime}(v), \alpha(v)$ and $C_{\text {ID }}^{v^{2}}(v)$ are the imaginary part of the complex refractive index, the absorption coefficient and IR-vibration coupling constant, respectively.

For Raman scattering intensity $I_{\text {raw }}(v)$, the boson peak is given by,

$\frac{\chi^{\prime \prime}(v)}{v}=\frac{I_{\text {raw }}(v)}{v \cdot[n(v)+1]}=C_{R}(v) \cdot \frac{g(v)}{v^{2}}$

where $\mathrm{C}_{\mathrm{R}}(v), n(v)$ are the Raman-vibration coupling constant and the Bose-Einstein factor, respectively.

The broad peak has been observed also in the temperature dependence of $C_{p} / T^{3}$ curve at low temperatures, where $C_{p}$ is the heat capacity. It has been known that the boson peak frequency has the strong correlation with the shear modulus [29], and the molecular dynamical simulation predicted recently that the origin of the boson peak is the Ioffe-Regal limit of a transverse acoustic mode, and its frequency is proportional to shear modulus [30].

Figure 6 the imaginary parts of the dielectric constant and Raman susceptibility divided by squared frequency for the glassy IMC at 100 $\mathrm{K}$. The broad peaks in two spectra are assigned to boson peaks with the peak frequencies 0.3 and $0.5 \mathrm{THz}$ for THz-TDS and Raman scattering measurements, respectively. The difference in peak frequencies is attributed to the different frequency dependence in between IRvibration and Raman-vibration coupling constants. The intermediate correlation length of the glassy structure was estimated to be about 2.5 $\mathrm{nm}[20]$.

For the characterization of polymorphic and polyamorphic pharmaceuticals, it is very important to measure both THz-TDS and low-frequency Raman scattering. The two methods have the complimentary selection rules which enable the full information on vibrational properties related to the crystalline structure, glassy intermediate range order, and their strength of intermolecular interactions.

\section{Conclusion}

We report the review on the application of the broadband THz-TDS and low-frequency Raman scattering spectroscopy to evaluate vibrational properties of glassy and crystalline pharmaceuticals. The real and imaginary parts of complex dielectric constants were determined by the transmission of THz-TDS using pure pellets without mixing polyethylene in the frequency range from 0.2 to $6.5 \mathrm{THz}$. The low-frequency Raman spectra were measured down to $0.3 \mathrm{THz}$ using a double-grating monochromator. In crystalline indomethacin, indapamide, and racemic ketoprofen, the clear difference in peak frequencies was observed between $\mathrm{THz}$ dielectric and low-frequency Raman scattering spectra. It can be attributed to the mutual exclusion principle between Raman and IR activities of optical vibrational modes in centrosymmetric crystals. For glassy indomethacin, the boson peak, which is the characteristic low-energy excitation of glassy and amorphous materials, was clearly observed at about $0.3 \mathrm{THz}$ and $0.5 \mathrm{THz}$ in THz-TDS and Raman scattering spectra, respectively. The difference in peak frequencies is attributed to the different frequency dependence in between IR-vibration and Raman-vibration coupling constants. In glassy indomethacin the broad peak at $3.0 \mathrm{THz}$ in the IR

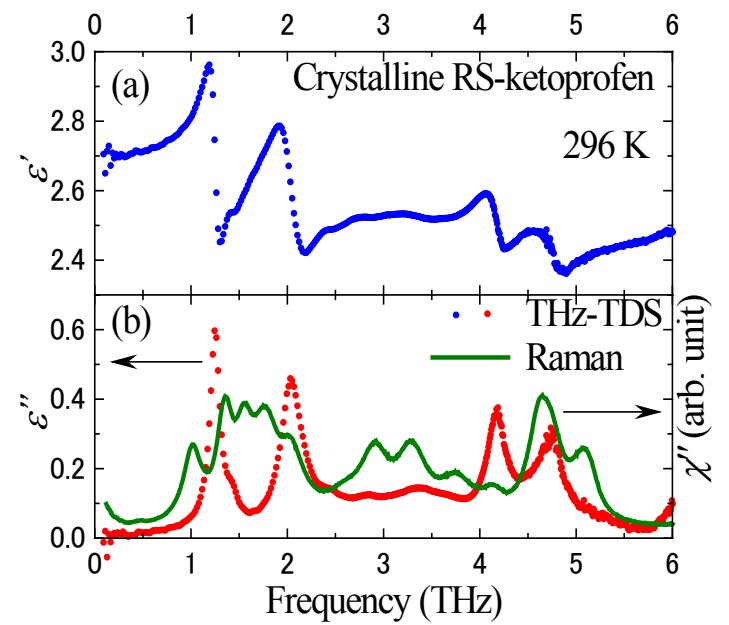

Figure 3: (a) Real part of the complex dielectric constant, $\varepsilon^{\prime}(v)$, and (b) the imaginary parts of the complex dielectric constant, $\varepsilon$ " $(v)$, and the Raman susceptibility, X'(v), of the commercial type crystalline RS ketoprofen at room temperature.

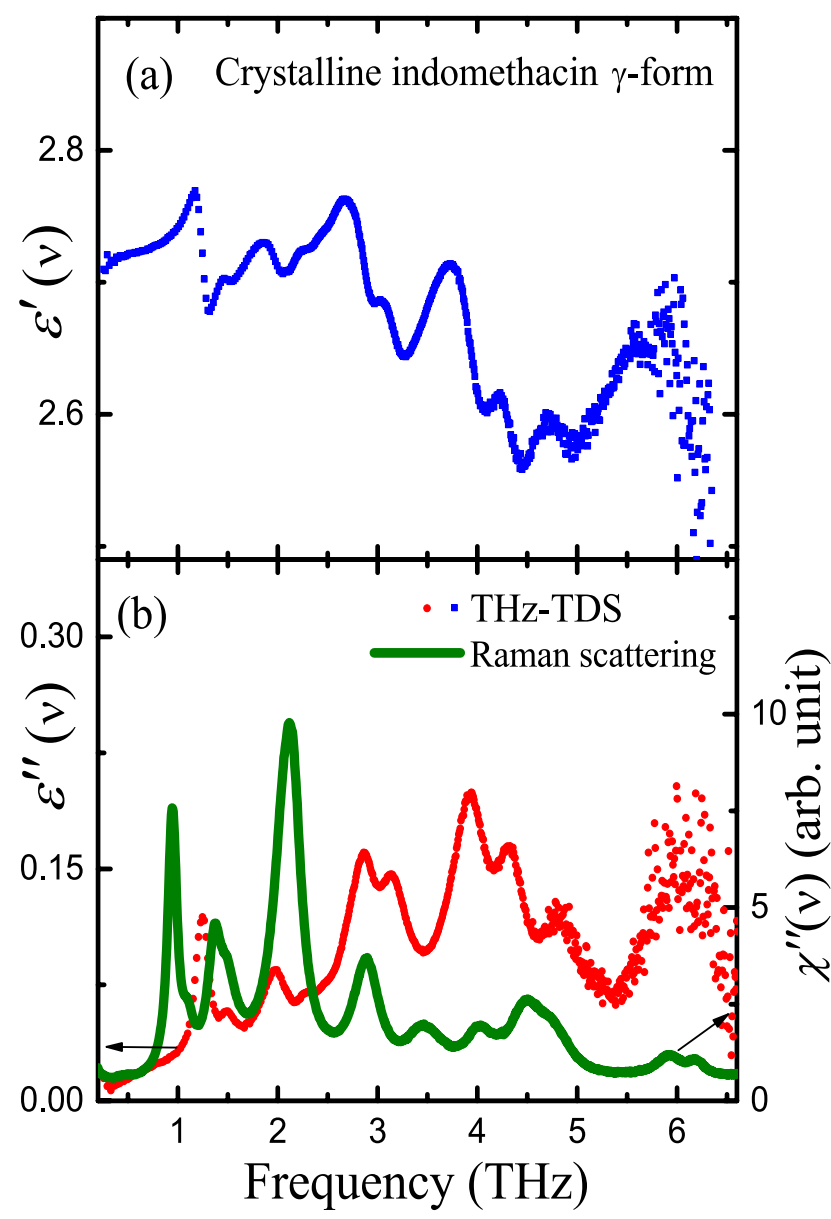

Figure 4: (a) The real part of the complex dielectric constant, $\varepsilon^{\prime}(\mathrm{v})$, and (b) the imaginary parts of the complex dielectric constant, $\varepsilon$ " $(v)$, and the Raman susceptibility, $X "(v)$, of the commercial type crystalline state of $\gamma$-form indomethacin at room temperature. 
Citation: Kojima S, Mori T, Shibata T, Kobayashi Y (2015) Broadband Terahertz Time-Domain and Low-Frequency Raman Spectroscopy of Crystalline and Glassy Pharmaceuticals. Pharm Anal Acta 6: 401. doi:10.4172/21532435.1000401

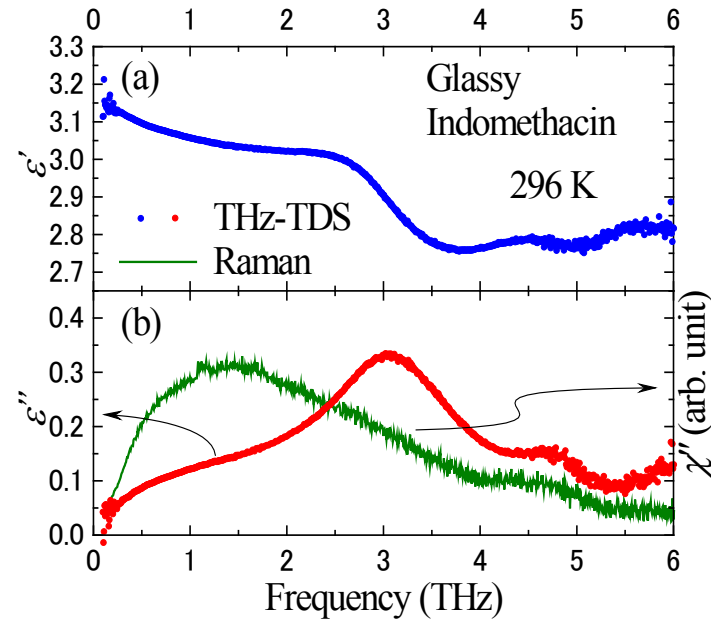

Figure 5: (a) The real part of the complex dielectric constant, $\varepsilon^{\prime}(v)$, and (b) the imaginary parts of the complex dielectric constant, $\varepsilon$ " $(v)$, and the Raman susceptibility, X" (v), of a glassy indomethacin at room temperature.

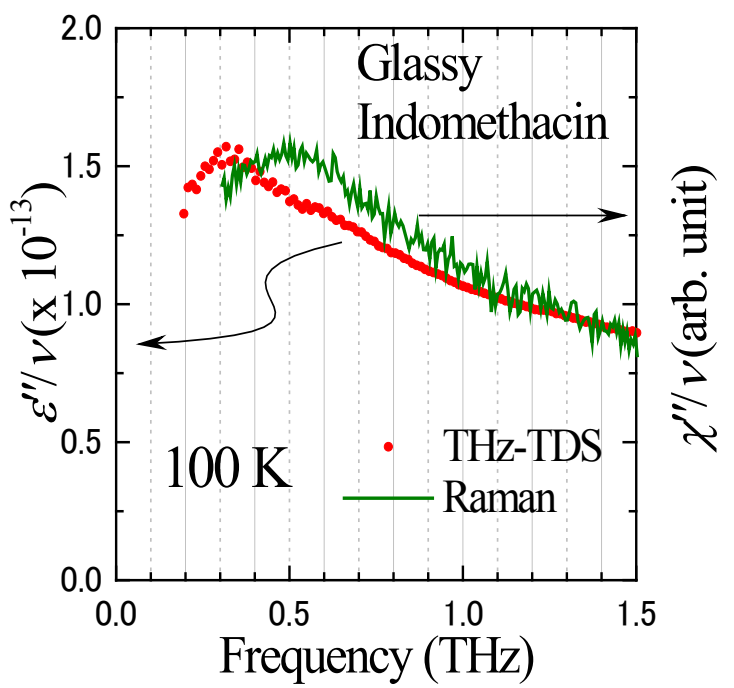

Figure 6: The imaginary parts of the complex dielectric constant, $\varepsilon^{\prime}(v)$, and the Raman susceptibility, $X "(v)$, divided by frequency $v$ of glassy indomethacin at room temperature.

spectrum can be attributed to the infrared active intermolecular vibrational mode of the hydrogen bonded cyclic dimers. For the study of polymorphic and polyamorphic pharmaceuticals, the simultaneous measurement of broadband THz-TDS and low-frequency Raman scattering spectroscopy is very important to give new insights into their structure and dynamics.

\section{Acknowledgements}

This work was supported in part by the JSPS KAKENHI Grant Numbers 90134204 and 24740194, and the Sasakawa Scientific Research Grant. Authors are grateful for the technical support on THz-TDS measurements to Sendai Office, Advantest Corporation.

\section{References}

1. Parrott EPJ, Zeitler JA (2015) Terahertz Time-Domain and Low-Frequency Raman Spectroscopy of Organic Materials. Appl Spec 69: 1-25.
2. Kužel $P$, Kadlec $F$ (2008) Tunable structures and modulators for THz light. C R Physique 9: 197-214.

3. Kojima S, Tsumura N, Wada Takeda M, Nishizawa S (2003) Far-infrared phonon-polariton dispersion probed by terahertz time-domain spectroscopy. Phys Rev B 67: 0351024-1-5.

4. Shibuya T, Suizu K, Kawase K (2010) Widely Tunable Monochromatic Cherenkov Phase-Matched Terahertz Wave Generation from Bulk Lithium Niobate. Appl Phys Express 3: 082201-1-3.

5. Mori T, Igawa H, Kojima S (2014) Progress of ultrafast terahertz time-domain spectroscopy: Raman inactive soft mode in quantum paraelectric $\mathrm{SrTiO}_{3}$. IOP Conf Ser Mater Sci Eng 54: 012006-1-5.

6. Novikov VN, Surovtsev NV, Wiedersich J, Adichtchev S, Kojima S, et al. (2002) On the origin of quasi-elastic light scattering in glasses. Europhys Lett 57 : 838-844.

7. Maczka M, Knyazev AV, Majchrowski A, Hanuza J, Kojima S (2012) Temperature-dependent Raman scattering study of the defect pyrochlores $\mathrm{RbNbWO}_{6}$ and $\mathrm{CsTaWO}_{6}$. J Phys: Condens Matter 24: 195902-1-10.

8. Wojnarowska Z, Grzybowska K, Hawelik L, Dulski M, Wrzalik R, et al. (2013) Molecular Dynamics, Physical Stability and Solubility Advantage from Amorphous Indapamide Drug. Mol Pharmaceutics 10: 3612-3627.

9. Ghugare P, Dongre V, Karmuse P, Rana R, Singh D, et al. (2010) Solid state investigation and characterization of the polymorphic and pseudopolymorphic forms of indapamide. J Pharm Biomed Analy 51: 532-540.

10. Smrkolj M, Meden A (2006) Crystal structure of indapamide determined from powered diffraction data. Pharmazie 61: 999-1004

11. Kobayashi Y, Shibata T, Mori T, Kojima S (2015) Terahertz Time-Domain Spectroscopy and Low-Frequency Raman Scattering of Crystalline and Glassy Pharmaceutical Indapamide. Int Lett Chem Phys Astro 7: 46-22.

12. Kojima S, Shibata T, Igawa H, Mori T (2014) Broadband terahertz time-domain spectroscopy: Crystalline and glassy drug materials. IOP Conf Ser: Mater Sci Eng 54: 012001-1-6.

13. Honglu Y, Ching CB (2004) Physicochemical properties, binary and ternary phase diagrams of ketoprofen. Chirality 16: 541-548.

14. Vueba ML, Pina ME, Veiga F, Sousa JJ, de Carvalho LA (2006) Conformationa study of ketoprofen by combined DFT calculations and Raman spectroscopy. Int J Pharm 307: 56-65.

15. Shibata T, Igawa H, Kim TH, Mori T, Kojima S (2014) Glass transition dynamics of anti-inflammatory ketoprofen studied by Raman scattering and terahertz time-domain spectroscopy. J Mol Struc 1062: 185-188.

16. Strachan CJ, Rades T, Newnham DA, Gordon KC, Pepper M, et al. (2004) Using terahertz pulsed spectroscopy to study crystallinity of pharmaceutical materials. Chem Phys Lett 390: 20-24.

17. Strachan CJ, Taday PF, Newnham DA, Gordon KC, Zeitler JA, et al. (2005) Using terahertz pulsed spectroscopy to quantify pharmaceutical polymorphism and crystallinity. J Pham Sci 94: 837-846.

18. Otsuka M, Nishizawa J, Fukura N, Sasaki T (2012) Characterization of PolyAmorphous Indomethacin by Terahertz Spectroscopy. J Infrared Milli Terahz Waves 33: 953-962.

19. Kistenmacher TJ, Marsh RE (1972) Crystal and molecular structure of an anti-inflammatory agent, indomethacin, 1-(p-chlorobenzoyl)-5-methoxy-2methylindole-3-acetic acid. J Am Chem Soc 94: 1340-1345.

20. Shibata T, Mori T, Kojima S (2015) Low-frequency vibrational properties of crystalline and glassy indomethacin probed by terahertz time-domain spectroscopy and low-frequency Raman scattering. Spectrochimica Acta Part A: Molecular and Biomolecular Spectroscopy 150: 207-211.

21. Ike Y, Seshimo Y, Kojima S (2009) Crystallization and vitrification of cryoprotectants studied by Raman scattering, Brillouin scattering and $\mathrm{THz}$ TDS. J Mol Struct 924-926: 127-130.

22. Yomogida Y, Sato Y, Nozaki R, Mishina T, Nakahara J (2010) Dielectric study of normal alcohols with $\mathrm{THz}$ time-domain spectroscopy. Journal of Molecula Liquids 154: 31-35.

23. Rønne C, Åstrand PO, Keiding SR (1999) THz Spectroscopy of Liquid $\mathrm{H}_{2} \mathrm{O}$ and $\mathrm{D}_{2} \mathrm{O}$. Phys Rev Lett 82: 2888-2891. 
Citation: Kojima S, Mori T, Shibata T, Kobayashi Y (2015) Broadband Terahertz Time-Domain and Low-Frequency Raman Spectroscopy of Crystalline and Glassy Pharmaceuticals. Pharm Anal Acta 6: 401. doi:10.4172/21532435.1000401

24. Carpentier L, Decressain R, Despre S, Descamps M (2006) Dynamics of the Amorphous and Crystalline $\alpha-$, $Y$-Phases of Indomethacin. J Phys Chem B 110: 457-464

25. Wojnarowska Z, Adrjanowicz K, Wlodarczyk P, Kaminska E, Kaminski K, et al. (2009) Broadband Dielectric Relaxation Study at Ambient and Elevated Pressure of Molecular Dynamics of Pharmaceutical: Indomethacin. J Phys Chem B 113: 12536-12545.

26. Jakobsen RJ, Mikawa Y, Brasch JW (1967) Far infrared studies of hydrogen bonding in carboxylic acids-I formic and acetic acids. Spectrochim Acta 23: 2199-2209.
27. Jakobsen RJ, Mikawa Y, Brasch JW (1968) Far infrared studies of hydrogen bonding in carboxylic acids-II: The n-alkyl acids, propanoic to undecanoic. Spectrochim Acta 25: 839-847.

28. Kojima S (1993) Low-frequency Raman Inverstigation of the liquid-glass transition in glycerol. Phys Rev B 47: 2924-2927.

29. Kojima S, Matsuda Y, Fukawa Y, Kawashima M, Moriya Y, et al. (2010) Boson peaks of lithium borate glasses studied by inelastic neutron and light scattering J Non-Crys Solids 356: 2524-2527.

30. Shintani H, Tanaka H (2008) Universal link between the boson peak and transverse phonons in glass. Nat Mater 7: 870-877. 